\title{
Correlação entre o risco de queda e autonomia funcional em idosos institucionalizados
}

\author{
Correlation between the risk of falling and functional autonomy in institutionalized elderly
}

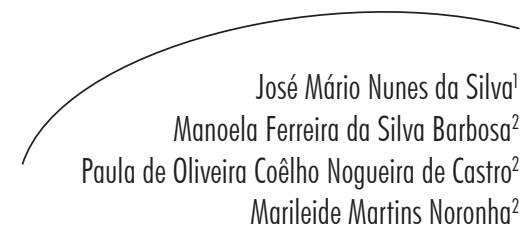

\section{Resumo}

Uma importante consequência do envelhecimento é o aumento do número de idosos com dependência funcional e propensão a quedas. Esta pesquisa teve por objetivo correlacionar o risco de quedas com a autonomia funcional em idosos institucionalizados na cidade de Teresina-PI. O modelo de estudo adotado foi observacional, descritivo e transversal, tendo sido realizado em três instituições de longa permanência, com 47 idosos. Os instrumentos da pesquisa foram a escala de equilíbrio de Berg (EEB) e o índice de Katz. Observou-se que o gênero feminino apresentou pior desempenho funcional médio quando comparado ao masculino (34,95 \pm 16,22 vs 47,07 \pm 11,67; $p<0,01)$ e houve correlação positiva e forte $(\mathrm{r}=0,735$ e $p<0,001)$ entre os escores da EEB e o índice de Katz. Houve forte correlação entre as duas escalas no estudo, ou seja, quanto melhor o resultado da escala de equilíbrio de Berg, melhor o desempenho nas atividades básicas de vida diária e vice-versa.

\section{Abstract}

An important consequence of aging is the increase of functional dependency and risk of falls in elderly. This study had an observational, descriptive, cross-sectional design and aimed to correlate the risk of falls with the functional autonomy in the institutionalized elderly in the city of Teresina, state of Piaui, Brazil. It was conducted in three long-term institutions, with 47 seniors. The research instruments were the Berg balance scale (BBS) and Katz index. It was observed that females had a worse average functional performance when compared to males $(34.95 \pm 16.22$ vs $47.07 \pm 11.67$; $\mathrm{p}<0.01)$ and positively and strongly correlated between the scores of BSE and the Katz index $(r=0.735$ and $\mathrm{p}<0.001)$. There was strong correlation between the two scales in the study, ie, the better the result of the Berg balance scale, the best performance in basic activities of daily living and vice versa.

\footnotetext{
Programa de Pós-graduação em Ciências e Saúde. Universidade Federal do Piauí. Teresina, PI, Brasil.

Departamento de Fisioterapia. Centro de Ensino Unificado de Teresina. Teresina, PI, Brasil.

Palavras-chave: Limitação da Mobilidade. Equilíbrio Postural. Idoso. Saúde do Idoso Institucionalizado. Risco de Quedas.

Key words: Mobility Limitation. Postural Balance. Aged. Health of Institutionalized Elderly. Risk of Falls. 


\section{INTRODUÇÃO}

O crescimento da população idosa é um fenômeno mundial e, no Brasil, as modificações se dão de forma radical e bastante acelerada. As projeções mais conservadoras indicam que, em 2020, o país já será o sexto do mundo em número de idosos, com um contingente superior a 30 milhões de pessoas. ${ }^{1}$

Embora esse crescimento seja um importante indicativo da melhoria da qualidade de vida, é bem conhecido que o processo de envelhecimento está atrelado a perdas importantes em inúmeras capacidades físicas, as quais culminam, inevitavelmente, no declínio da capacidade funcional e da independência do idoso para atividades cotidianas conhecidas como atividades básicas de vida diária. ${ }^{2-4}$

O risco de cair aumenta significativamente com o avançar da idade, pois o envelhecimento traz várias alterações anatômicas e fisiológicas, tornando o idoso mais frágil e mais propenso a sofrer quedas. ${ }^{5}$

A queda pode ser definida como um deslocamento não intencional do corpo para um nível inferior à posição inicial, com incapacidade de correção em tempo hábil. Afirma-se ainda que as quedas podem ser manifestações de patologias existentes, resultantes da interação entre diversos fatores que comprometem a estabilidade do indivíduo, tais como: fatores ambientais, fisiológicos, psicossociais e biomédicos. ${ }^{6,7}$

Para os idosos, as quedas possuem um significado muito relevante, pois podem leválos a incapacidade e morte. Seu custo social é imenso e torna-se maior quando o idoso tem diminuição da autonomia e da independência ou passa a necessitar de institucionalização. ${ }^{8}$ Devido às complicações para a saúde, os custos assistenciais e sua alta incidência, as quedas em idosos são consideradas como um dos principais problemas de saúde pública. ${ }^{6,9}$ Elas representam as principais causas de acidentes em idosos. ${ }^{8}$

Os principais fatores predisponentes encontrados na maioria dos estudos são: idade igual ou superior a 75 anos, sexo feminino, presença de declínio cognitivo, inatividade, fraqueza muscular e distúrbios do equilíbrio corporal, marcha ou mobilidade, quedas anteriores com ou sem fraturas, comprometimento para realizar atividades de vida diária e o uso de medicamentos, entre outros..$^{6-9}$

Existem ainda fatores sociodemográficos, como a institucionalização, que representa um fator de risco para quedas por denotar um caráter de atenção e suporte a idosos fragilizados na grande maioria. Mesmo que o idoso institucionalizado não esteja fragilizado por algum distúrbio orgânico, muitas vezes o isolamento, o abandono e o grau de inatividade física contribuem para a propensão a quedas, por acelerar o curso do envelhecimento. ${ }^{10-12}$

Entre os instrumentos para avaliar a funcionalidade dos idosos, destaca-se o índice de Katz, ${ }^{13}$ o qual possui seis itens que são hierarquicamente relacionados e refletem os padrões de desenvolvimento infantil, ou seja, que a perda da função no idoso começa pelas atividades mais complexas, como vestir-se, banhar-se, até chegar as de autorregulação, como alimentar-se, e as de eliminação ou excreção. ${ }^{14}$

Para Lajoie \& Gallagher, ${ }^{9}$ a escala de equilíbrio de Berg é um teste que apresenta elevados índices de sensibilidade e especificidade, podendo ser considerado valioso para avaliação e previsão de futuras quedas. A presente pesquisa, portanto, tem como objetivo correlacionar o risco de quedas com a autonomia funcional em idosos institucionalizados na cidade de Teresina-PI.

\section{METODOLOGIA}

O presente estudo consistiu de uma pesquisa descritiva, observacional com característica transversal, direta e de natureza quantitativa. A pesquisa foi realizada no período de agosto a outubro de 2011, em três instituições de longa permanência de Teresina-PI: Fundação Abrigo São Lucas, Vila do Ancião e Casa Frederico Ozanam. 
Foi aprovada pelo Comitê de Ética e Pesquisa do Centro de Ensino Unificado de Teresina, sob o $\mathrm{n}^{\mathrm{o}}$ de protocolo 7.990/2011, e seguiu as recomendações da Resolução n ${ }^{\circ}$ 196/96 do Conselho Nacional de Saúde. Todos os participantes da pesquisa assinaram o Termo de Consentimento Livre e Esclarecido (TCLE), concordando com a participação.

Aamostra foidotipoaleatório porconveniência e constituiu-se de 47 idosos de ambos os gêneros que aceitaram, por livre e espontânea vontade, participar da pesquisa. Foram adotados como critérios de exclusão: (1) presença de incapacidade funcional (restrito ao leito, usuário de cadeira de rodas); (2) comprometimento cognitivo que os incapacitem para a compreensão e execução de comandos; (3) deficientes visuais; (4) idosos que apresentem sinais vitais que coloquem em risco sua saúde durante a realização do teste.

A coleta de dados foi realizada em horários estabelecidos pelos responsáveis de cada instituição. Utilizaram-se uma ficha semiestruturada produzida pelos autores, a escala de equilíbrio de Berg e o índice de Katz para atividades básicas de vida diária (ABVDs).

Logo após o preenchimento da ficha de coleta de dados, foi aplicada a escala de equilíbrio de Berg, que avalia o desempenho do equilíbrio funcional em 14 itens comuns da vida diária. Cada item possui uma escala ordinal de cinco alternativas que variam de zero a quatro pontos. Os pontos são baseados no tempo em que uma posição pode ser mantida, na distância em que o membro superior é capaz de alcançar à frente do corpo e no tempo para completar uma determinada tarefa. O procedimento foi realizado de forma individual e única com cada paciente, tendo duração de 25 a 30 minutos. Os escores obtidos foram pontuados no final da avaliação, onde a pontuação máxima é de 56 pontos. ${ }^{15}$

Aplicou-se o índice de Katz, ${ }^{13}$ desenvolvido para pacientes institucionalizados e frequentemente utilizado para a avaliação das ABVDs em idosos. O grau de assistência exigido é avaliado em seis atividades: tomar banho, vestir-se, ir ao banheiro, transferência, continência e alimentar-se. $O$ índice de Katz foi aplicado no formato Likert, que pontua cada item de 0 a 3 , sendo que a pontuação 0 representa independência completa; 1 está relacionada com o uso de ajuda não humana (acessórios como bengalas, barras, apoio em móveis); 2 relaciona-se a ajuda humana, e a pontuação 3, uma completa dependência.

O processamento dos dados e a análise estatística foram realizados por meio do programa SSPSS ${ }^{\circledR}$, versão 18.0 para Windows. Os dados foram analisados primeiramente pelo teste de Shapiro-Wilk, para avaliar a normalidade. Para verificar diferença entre as médias, utilizouse o teste $U$ de Mann Whitney. A associação entre as variáveis foi realizada pelo teste quiquadrado. O risco de quedas foi calculado por meio da razão de chance (odds ratio) com seu respectivo IC95\%. Para classificar o risco de queda, utilizou-se o ponto de corte proposto por Berg et al., ${ }^{16}$ em que escores abaixo de 45 são preditivos de futuras quedas.

Para correlacionar as variáveis do estudo, foi aplicada a correlação de Spearman. Em todos os testes aplicados, considerou-se sempre um nível de significância estatística de 95\%, ou seja, p<0,05.

\section{RESULTADOS}

Os sujeitos da pesquisa foram 47 idosos, sendo que $60 \%$ são do sexo masculino $(\mathrm{n}=28)$ e $40 \%$ do sexo feminino ( $\mathrm{n}=19)$; a média de idade encontrada foi de 70,7 $\pm 9,2$, com mediana de 69 anos, onde a maioria dos idosos se encontrava na faixa de 60 e 79 anos e não praticava nenhum tipo de atividade física e 89,4\% ( $\mathrm{n}=42$ ) fazem uso de algum tipo de medicamento.

$\mathrm{Na}$ presente pesquisa, não houve evidências de que idosos de uma determinada instituição estivessem mais propensos a sofrer quedas que os demais, de acordo com o teste qui-quadrado, que revelou um valor de $\mathrm{p}>0,05(\mathrm{p}=0,243)$. Dessa forma, não foi necessário separar os idosos por instituições para análise dos resultados referentes às quedas. 
O desempenho médio do equilíbrio funcional do sexo masculino foi $47,07 \pm 11,67$ e, no sexo feminino, 34,95 $\pm 16,22(\mathrm{p}<0,01)$, revelando uma diferença muito significativa nos escores da escala de equilíbrio de Berg entre os gêneros. Entre os 28 idosos do sexo masculino, 23 obtiveram escore superior a 45 pontos (pontuação de corte); e dos 18 idosos do sexo feminino, apenas sete tiveram escore superior a 45 pontos (tabela 1).
Ao se avaliar os escores médios do índice de Katz, nota-se que o sexo masculino obteve valores

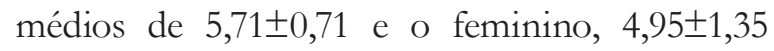
$(\mathrm{p}<0,05)$, ou seja, houve diferença significativa nos escores da escala. Entre os 28 idosos do sexo masculino, apenas cinco obtiveram escore inferior a seis e maior que quatro, o que denota uma dependência parcial; já dos 19 do sexo feminino, apenas oito tiveram escores superiores a cinco.

Tabela 1 - Dados comparativos em média da escala de equilíbrio de Berg e índice de Katz, divididos por gênero ( $\mathrm{n}=47)$. Teresina-PI, 2011.

\begin{tabular}{|c|c|c|c|c|}
\hline \multirow{2}{*}{ Variáveis } & Masculino $(\mathrm{n}=28)$ & Feminino $(n=19)$ & \multirow{2}{*}{$P$} & Total \\
\hline & Média \pm DP & Média \pm DP & & Média \pm DP \\
\hline Escala de Berg & $47,07 \pm 11,67$ & $34,95 \pm 16,22$ & $0,002 b$ & $42,17 \pm 14,80$ \\
\hline Índice de Katz & $5,71 \pm 0,71$ & $4,95 \pm 1,35$ & $0,021 \mathrm{a}$ & $5,40 \pm 1,08$ \\
\hline
\end{tabular}

${ }^{\mathrm{a}} p<0,05$ : diferença significativa; ${ }^{\mathrm{b}} p<0,01$ : diferença muito significativa.

O gráfico 1 mostra o risco de queda dos idosos. Nota-se que 47\% ( $\mathrm{n}=22)$ possuem maior risco de queda e 53\% ( $\mathrm{n}=25)$ possuem menor risco. Quando se analisa o risco de queda (expresso pela OR, odds ratio) entre os gêneros, encontra-se um valor de 3,90 com IC95\% de 1,31 a 13,45 e $\mathrm{p}<0,05$, ou seja, o sexo feminino no presente estudo aumenta em 3,9 vezes as chances de queda que o sexo masculino.

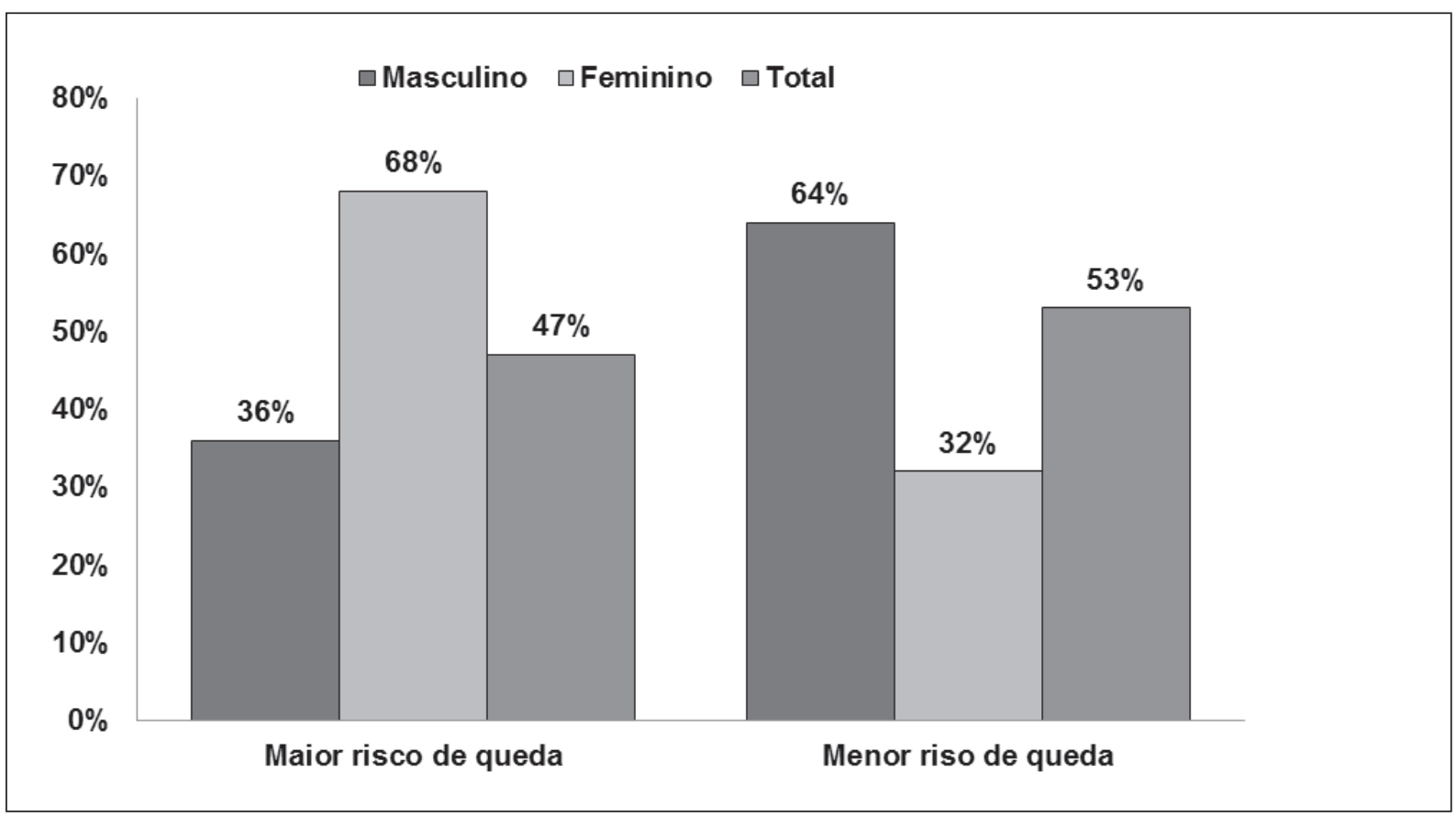

Ponto de corte na escala de Berg $=45 ; \mathrm{OR}=3,90, \mathrm{IC} 95 \%=1,31-13,45$ e $p=0,028$.

Gráfico 1 - Distribuição dos idosos segundo o risco ou não de quedas, de acordo com a escala de equilíbrio de Berg, divididos por gênero. Teresina-PI, 2011. 
O gráfico 2 mostra a capacidade funcional dos idosos para atividades básicas de vida diária, onde $70 \%(n=33)$ se encontram em dependência parcial e 30\% ( $n=14)$ são independentes nas atividades básicas de vida diária. Quando se avalia a capacidade funcional por gênero pela odds ratio, encontra-se um valor de 4,14 com IC95\% de 1,10 a 15,52 e p<0,05, ou seja, o sexo feminino no presente estudo aumenta em 4,14 vezes as chances de ser dependente parcial para as ABVDs que o masculino.

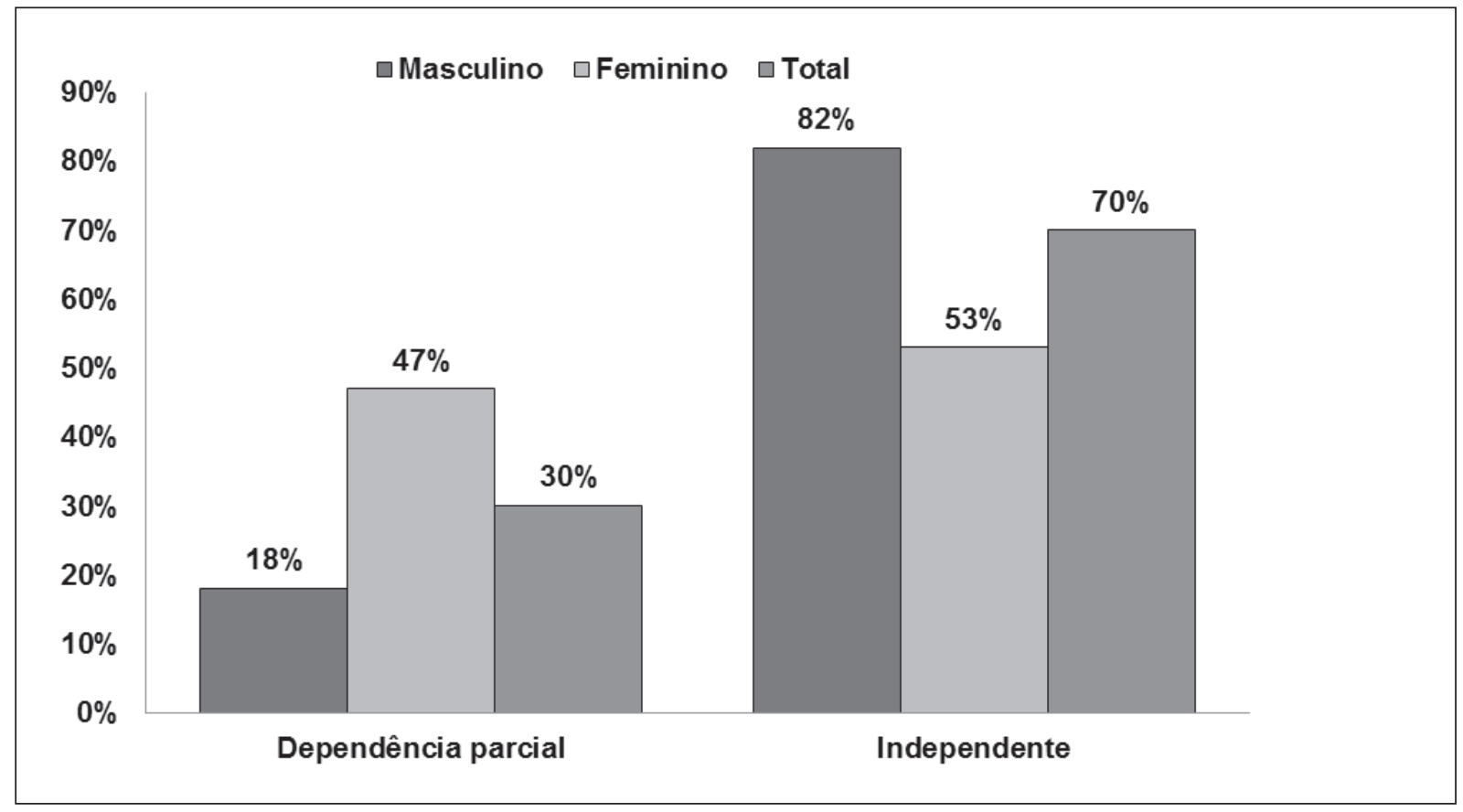

$\mathrm{OR}=4,14, \mathrm{IC} 95 \%=1,10-15,52$ e $p=0,030$.

Gráfico 2 - Distribuição dos idosos, segundo a escala de atividades básicas de vida diária. Teresina-PI, 2011.

Houve correlação positiva e forte $(\mathrm{r}=0,735 \mathrm{e}$ $\mathrm{p}<0,001)$ entre os escores da escala de equilíbrio de Berg e índice de Katz que avalia as ABVDs, ou seja, quanto melhor o resultado da escala de equilíbrio de Berg, melhor o desempenho nas atividades básicas de vida diária e vice-versa (gráfico 3). 


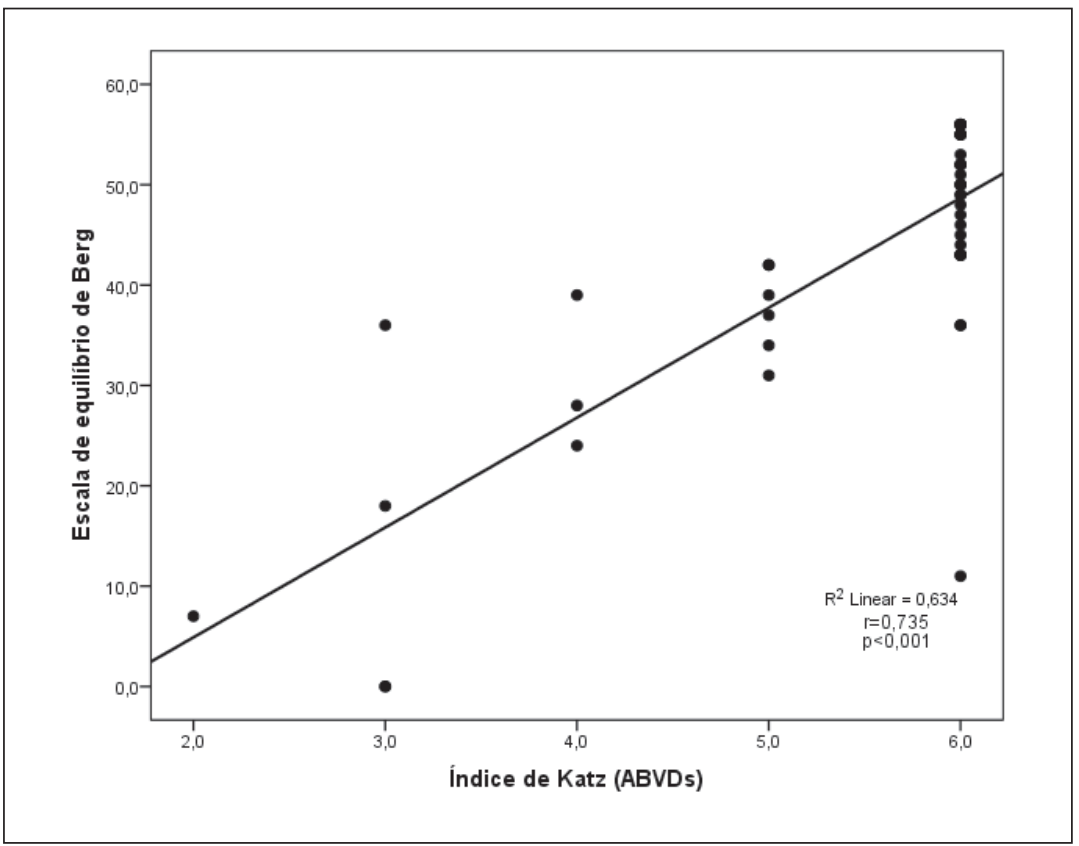

$\mathrm{r}=0,735$ (correlação positiva e forte entre as variáveis); $p<0,001$ (correlação extremamente significativa).

Gráfico 3 - Gráfico de dispersão comparativo entre a escala de equilíbrio de Berg e a escala de atividades básicas de vida diária (ABVDs). Teresina-PI, 2011.

\section{DISCUSSÃO}

Os dados obtidos nas três instituições pesquisadas mostram maior número de idosos do sexo masculino que o feminino. Segundo Nasri, ${ }^{17}$ no ano de 2000, para cada 100 mulheres idosas havia 81 homens idosos; em 2050 haverá provavelmente cerca de 76 idosos para 100 idosas.

Ueno et al. ${ }^{18}$ identificaram o sexo feminino e idade superior a 70 anos como alguns dos fatores mais relacionados a quedas. Perracini \& Ramos $^{6}$ concluíram em seus estudos que ser do sexo feminino aumenta as chances de quedas em 2,29 (IC95\% 1,70-3,076 e $\mathrm{p}<0,001$ ). Vellas et al. ${ }^{19}$ identificaram em sua pesquisa um risco de quedas de 1,33 associado ao sexo feminino (IC95\% 1,08-1,61 e p<0,001).

Outro fato observado na população estudada foi que todos os idosos eram sedentários. Estudo de Pimentel \& Scheicher ${ }^{20}$ confirma essa relação, mostrando que idosos sedentários têm 15,58 vezes (IC95\% 1,8-128,9 e $p=0,002)$ mais chances de quedas do que idosos ativos. Gardner,
Robertson \& Campbell $^{21}$ complementam explicando que, dentre as estratégias para diminuir a ação dos fatores de risco para queda em idosos, a prática de atividade física tem sido comprovada com uma proposta bastante eficaz. Pesquisas demonstram ainda que pessoas idosas se mostram mais propensas a sofrer quedas, o que pode ser justificado, em parte, pela perda de força progressiva decorrente da senescência. ${ }^{4}$

No presente estudo, o desempenho médio geral da escala de equilíbrio foi $42,17 \pm 14,80$ (tabela 1), denotando maior risco de queda da população estudada. Esses dados corroboram estudos de Wolf et al. ${ }^{22}$ e Zambaldi et al., ${ }^{23}$ que também encontraram valores médios da escala de equilíbrio abaixo do ponte de corte.

Gazzola etal.; ${ }^{24}$ Ruwer, Rossi\&Simon; ${ }^{25}$ Maciel \& Guerra ${ }^{26}$ enfatizam que o baixo desempenho funcional dos idosos pode ser explicado em parte pelo próprio processo natural do envelhecimento, que compromete a habilidade do sistema nervoso central de realizar o processamento dos sistemas vestibular, proprioceptivo e visual, 
alterando o controle postural, em virtude da diminuição na velocidade de condução das informações, gerando instabilidade e pondo em risco a movimentação segura dos idosos, por sua predisposição a quedas.

Outro fator importante que pode ter influenciado nos valores da escala no estudo é que todos os idosos participantes da pesquisa são institucionalizados, situação que já acarreta um declínio funcional, pois segundo Perracini \& Ramos, ${ }^{6}$ os idosos institucionalizados apresentaram menor mobilidade funcional que os idosos não institucionalizados, podendo, por decorrência, estar expostos a maior risco de quedas.

Quando se avaliou o desempenho funcional médio por gênero, observou-se que o sexo feminino teve pior desempenho médio $(34,95$ $\pm 16,22)$, quando comparado ao masculino $(47,07 \pm 11,67$ e $\mathrm{p}<0,01)$, segundo a tabela 1 , e maior risco a sofrer quedas (gráfico 1). Barbosa ${ }^{27}$ relata que o sexo feminino é mais propenso a limitações funcionais, por serem frequentemente portadoras de maior número de condições crônicas, tais como depressão, artrose, artrite, entre outras, comparadas aos homens, o que pode explicar o pior desempenho no presente estudo.

Rubenstein \& Josephson $^{28}$ afirmam que idosos com osteoartrite apresentam risco de quedas 2,4 vezes maior que indivíduos sem essa doença. Concluem que a avaliação do grau de autonomia funcional dos idosos e o relato de quedas são fatores determinantes para o desenvolvimento de estratégias preventivas, que impeçam a progressão de incapacidades e preservem a autonomia.

Perracine \& Ramos ${ }^{6}$ complementam argumentando que o sexo feminino possui menor quantidade de massa magra e força muscular menor que os homens, o que em parte explica a maior fragilidade e predisposição a sofrer quedas. Além disso, Fried et al. ${ }^{29}$ afirmam que as mulheres poderiam também estar mais expostas a fatores extrínsecos que causam sarcopenia, como uma inadequada ingesta nutricional, comparadas aos homens.

Em relação aos dados referentes ao índice de Katz, encontraram-se valores médios que classificam a população do estudo em dependentes parciais (tabela 2). O sexo feminino teve média menor quando comparado ao masculino e um maior risco de serem dependentes para as atividades de vida diária (gráfico 2). Von Strauss et al..$^{30} \mathrm{e}$ Hardy et al. ${ }^{31}$ afirmam que mulheres são mais afetadas em sua autonomia para atividades cotidianas que os homens, como consequência de uma maior fragilidade óssea que pode ser progressivamente incapacitante em grande parte de suas vidas.

As dificuldades ou incapacidades encontradas pelos idosos os deixam mais dependentes do auxílio de outras pessoas para tarefas simples como sair de casa sozinho, progredindo para as tarefas de autocuidado como tomar banho ou ir ao banheiro sozinho. O fato de se tornar dependente faz com que o idoso se sinta desvalorizado, o que pode resultar no desenvolvimento de problemas psicológicos e baixa qualidade de vida. ${ }^{32,33}$

A dependência por si só, entretanto, não constitui evento negativo. Em diferentes etapas da vida, a pessoa pode ou não ser dependente, temporária ou definitivamente. ${ }^{34} \mathrm{~A}$ dependência se configura mais relevante quando surge em decorrência de eventos ocorridos em fases mais avançadas da vida, como na velhice, e as atividades básicas de vida diária são afetadas por essa dependência. ${ }^{35}$

A presente pesquisa encontrou, ainda, forte correlação $(r=0,732$ e $p<0,001)$ entre a escala de equilíbrio de Berg e o índice de Katz para atividades básicas de vida diária (gráfico 3). Figliolino et al. ${ }^{36}$ relatam que o risco de queda é ocasionado pela diminuição das habilidades de atividade de vida diária e/ou falta de equilíbrio, corroborando a relação encontrada no estudo. Fabrício et al. ${ }^{8}$ complementam afirmando que a queda traz como consequência para o idoso o aumento de dificuldade e de dependência para realização das atividades de vida diária (AVD), 
sendo que as mais prejudicadas após a queda são: deitar/levantar-se da cama, caminhar em superfície plana e tomar banho.

Para Pimentel \& Scheicher ${ }^{20}$ e Mazo et al., ${ }^{2}$ as quedas e suas consequências, mais frequentes na população idosa, representam um problema na saúde dessa faixa etária, pois ao cair os idosos correm mais riscos de lesões. Além disso, um idoso que sofreu uma queda pode desenvolver o que se chama de "síndrome pós-queda", em que o impacto psicológico pode levar à diminuição das ABVDs.

Vale ressaltar que, durante a pesquisa, buscou-se uma população mais homogênea sem diferença quanto a idade, nível cognitivo e sem históricos de doenças que pudessem afetar diretamente o equilíbrio.

Uma das limitações da pesquisa foi a pequena população estudada; portanto, sugerese que outras pesquisas com uma amostra maior devem ser estimuladas e realizadas, para verificar diferenças ou similaridades nos achados deste estudo.

\section{REFERÊNCIAS}

1. Carvalho JAM, Garcia RA. O envelhecimento da população brasileira: um enfoque demográfico. Cad Saúde Pública 2003;19(3):725-33.

2. Mazo GZ, Liposcki DB, Ananda C, Prevê D. Condições de saúde, incidência de quedas e nível de atividade física dos idosos. Rev Bras Fisioter 2007;11(6):437-42.

3. Freitas EV, Py L, Néri AL, Cançado FAX, Gorzoni ML, Rocha SM, editores. Tratado de Geriatria e Gerontologia. Rio de Janeiro: Guanabara Koogan; 2002.

4. Rebelatto JR, Castro AP, Chan A. Quedas em idosos institucionalizados: características gerais, fatores determinantes e relações com a força de preensão manual. Acta OrtopBras 2007;15(3):151-4.

\section{CONCLUSÃO}

Os resultados do estudo permitem inferir que a maioria da população de idosos estudada possui propensão a maior risco de queda avaliado pela escala de equilíbrio de Berg e uma dependência parcial para atividades básicas de vida diária segundo o índice de Katz.

As mulheres tiveram maior risco para sofrer queda e ser dependentes parciais para as atividades básicas de vida diária que os homens. Houve ainda forte correlação entre as duas escalas no estudo, ou seja, quanto melhor o resultado da escala de equilíbrio de Berg, melhor o desempenho nas atividades básicas de vida diária e vice-versa.

É essencial, portanto, que as instituições que abrigam idosos tenham conhecimento do risco de queda e grau de dependência dos mesmos, no sentido de planejarem uma assistência de forma individual e agregar recursos que possibilitem manter esse idoso o mais ativo possível. Desta forma, será possível desenvolver atividades específicas aos diferentes processos de envelhecimento.

5. Simoceli L, Bittar RMS, Bottino MA, Bento RF. Perfil diagnóstico do idoso portador de desequilíbrio corporal: resultados preliminares. Rev Bras Otorrinolaringol 2003;69(6):772-7.

6. Perracini MR, Ramos LR. Fatores associados a quedas em uma coorte de idosos residentes na comunidade. Rev Saúde Pública 2002;36(6)709-16.

7. Guimarães LHCT, Galdino DCA, Martins FLM, Vitorino DFM, Pereira KL, Carvalho EM. Comparação da propensão de quedas entre idosos que praticam atividade física e idosos sedentários. Rev Neurocienc 2004;12(2):68-72.

8. Fabrício SCC, Rodrigues RAP, Costa Junior ML. Causas e consequências de quedas de idosos atendidos em hospital público. Rev Saúde Pública 2004;38(1):93-9. 
9. Lajoie Y, Gallagher SP. Predicting falls within the elderly community: comparison of postural sway, reaction time, the Berg balance scale and the Activities-specific Balance Confidence (ABC) scale for comparing fallers and non-fallers. Arch Gerontol Geriatr 2004;38(1):11-26.

10. Jamet M, Deviterne D, Gauchard GC, Vançon G, Perrin PP. Higher visual dependency increases balance control perturbation during cognitive task fulfilment in elderly people. Neurosci Lett 2004;359(1-2):61-4.

11. Oliveira DLC, Goretti LC, Pereira LSM. O desempenho de idosos institucionalizados com alterações cognitivas em atividades de vida diária e mobilidade: estudo piloto. Rev Bras Fisioter 2006;10(1):91-6.

12. Soares AV. Matos FM, Laus LH, Suzuki S. Estudo comparativo sobre a propensão de quedas em idosos institucionalizados e não-institucionalizados através do nível de mobilidade funcional. Fisioterapia Brasil 2003;4(1):12-6.

13. Katz S, Downs TD, Cash HR, Grotz RC. Progress in development of the index of ADL. Gerontologist 1970;10(1):20-30.

14. Duarte YAO, Andrade CL, Lebrão ML. O Índex de Katz na avaliação da funcionalidade dos idosos. Rev Esc Enferm USP 2007;41(2):317-25.

15. Miyamoto ST, Lombardi Júnior I, Berg KO, Ramos LR, Natour J. Brazilian version of the Berg balance scale. Braz J Med Biol Res 2004;37(9):1411-21.

16. Berg KO, Wood-Dauphinnee SL, Williams JI, Maki B. Measuring balance in the elderly: validation of an instrument. Can J Public Heath 1992;83(Suppl 2):S7-11.

17. Nasri F. O envelhecimento populacional. Einstein (São Paulo) 2008;6(Supl1):S4-6.

18. Ueno M, Kawai S, Mino T, Kamoshita H. Systematic review of fall-related factors among the housedwelling elderly in Japan. Nihon Ronen Igakkai Zasshi 2006;43(1):92-101.

19. Vellas JB, Wayne SJ, Garry PJ, Baumgartner RN. A two-year longitudinal study of falls in 482 community-dwelling elderly adults. J Gerontol A Biol Sci Med Sci 1998;53(4):M264-74.

20. Pimentel RM, Scheicher ME. Comparação do risco de queda em idosos sedentários e ativos por meio da escala de equilíbrio de Berg. Fisioter Pesqui 2009;16(1):6-10.

21. Gardner MM, Robertson MC, Campbell AJ. Exercise in preventing falls and fall related injuries in older people: a review of randomised controlled trials. $\mathrm{Br} \mathrm{J}$ Sports Med 2000;34(1)7-17.

22. Wolf B, Feys H, De Weerdt, van der Meer J, Noom M, Aufdemkampe G. Effect of a physical therapeutic intervention for balance problems in the elderly: a single-blind, randomized, controlled multicentre trial. Clin Rehabil 2001;15(6):624-36.

23. Zambaldi PA, Costa TABN, Diniz GCLM, Scalzo PL. Efeito de um treinamento de equilíbrio em um grupo de mulheres idosas da comunidade: estudo piloto de uma abordagem específica não sistematizada e breve. Acta Fisiátrica 2007;14(1):17-24.

24. Gazzola JM, Perracini MR, Ganança MM, Ganança FF. Fatores associados ao equilíbrio funcional em idosos com disfunção vestibular crônica. Rev Bras Otorrinolaringol 2006;72(5):683-90.

25. Ruwer SL, Rossi AG, Simon LF. Equilíbrio no idoso. Rev Bras Otorrinolaringol 2005;71(3):298-303.

26. Maciel ACC, Guerra RO. Influência dos fatores biopsicossociais sobre a capacidade funcional de idosos residentes no nordeste do Brasil. Rev Bras Epidemiol 2007;10(2):178-89.

27. Barbosa AR, Souza JM, Lebrão ML, Laurenti R, Marucci MFN. Functional limitations of Brazilian elderly by age and gender differences: data from SABE Survey. Cad Saúde Pública 2005;21(4):1177-85.

28. Rubenstein LZ, Josephson KR. Risk factors for falls: a central role in prevention. J Am Soc Aging 2003;26(4):15-21.

29. Fried LP, Tangen CM, Walston J, Newman AB, Hirsh C, Gottdiener J, et al. Frailty in older adults: evidence for a phenotype. J Gerontol A Biol Sci Med Sci 2001;56(3):M146-56.

30. von Strauss E, Agüero-Torres H, Kareholt I, Winblad B, Fratiglioni L. Women are more disabled in basic activities of daily living than men only in very advanced ages: a study on disability, morbidity, and mortality from the Kungsholmen Project. J Clin Epidemiol 2003; 56(7):669-77.

31. Hardy SE, Allore HG, Guo Z, Gill TM. Explaining the effect of gender on functional transitions in older persons. Gerontology 2008;54(2):79-86.

32. Borges MRD, Moreira AK. Influências da prática de atividades físicas na terceira idade: estudo comparativo dos níveis de autonomia para o desempenho nas AVDs e AIVDs entre idosos ativos fisicamente e idosos sedentários. Motriz Rev Educ Fís 2009;15(3):562-73. 
33. Ozcan A, Donat H, Gelecek N, Ozdirenc M, Karadibak D. The relationship between risk factors for falling and the quality of life in older adults. BMC Public Health 2005;5:90.

34. Roper N, Logan W, Tierney AJ. O modelo de enfermagem Roper-Logan-Tierny: baseado nas atividades de vida diária. Lisboa: Climepsi; 2001.
35. Silva A, Almeida GJM, Casilhas RC, Cohen M, Peccin MS, Tufik S, et al. Equilíbrio, coordenação e agilidade de idosos submetidos à prática de exercícios físicos resistidos. Rev Bras Med Esporte 2008;14(2):88-93.

36. Figliolino JAM, Morais TB, Berbel AM, Dal Corso $\mathrm{S}$. Análise da influência do exercício físico em idosos com relação a equilíbrio, marcha e atividade de vida diária. Rev Bras Geriatr Gerontol 2009;12(2):227-38. 